\title{
Effect of Daily Chlorhexidine Bathing on Reducing Infections Caused by Multidrug-Resistant Organisms in Intensive Care Unit Patients: A Semi- Experimental Study with Parallel Controls
}

\section{Wenzhi Huang}

West China Hospital, Sichuan University https://orcid.org/0000-0002-4074-8422

\section{Fu Qiao}

Sichuan University West China Hospital

\section{Lin Cai}

Sichuan University West China Hospital

Zhiyong Zong ( $\nabla$ zongzhiyong@gmail.com )

West China Hospital, Sichuan University https://orcid.org/0000-0002-7675-260X

\section{Wei Zhang}

Sichuan University West China Hospital

\section{Research}

Keywords: $2 \%$ chlorhexidine gluconate, daily bathing, ICU, multidrug-resistant organisms

Posted Date: September 4th, 2020

DOI: https://doi.org/10.21203/rs.3.rs-67636/v1

License: (c) (i) This work is licensed under a Creative Commons Attribution 4.0 International License.

Read Full License 


\section{Abstract}

Background: A number of studies have shown that daily bathing by chlorhexidine (CHG) wipes in ICU can reduce healthcare-associated infections (HAls) caused by methicillin-resistant Staphylococcus aureus (MRSA) and vancomycin-resistant Enterococci (VRE). However, the impact of CHG bathing on carbapenem-resistant Acinetobacter baumannii (CRAB), carbapenem-resistant Pseudomonas aeruginosa (CRPA), and carbapenem-resistant Enterobacteriaceae (CRE) is not clear and remains controversial.

Methods: A semi-experimental study which employs both pre-controls and a parallel control was conducted. In the intervention period (from July 1 to December 31, 2016), strengthened infection control measures and daily bathing with $2 \%$ CHG-impregnated wipes once daily was performed in the ICU. Fiftyseven non-ICU wards with the occurrence of multidrug-resistant organisms (MDRO) infections during the same time period were selected as parallel control group (only CHG bathing was not performed). The net effect of the $2 \%$ CHG daily bathing was evaluated by the difference in difference (DID) model.

Results: The incidence of HAls caused by CRPA in ICU was significantly decreased between the intervention and pre-intervention period (2.5, 95\% confidence interval $(\mathrm{Cl}) 1.6-3.8$ vs. $4.6,95 \% \mathrm{Cl} 3.3-6.3$ cases $/ 1,000$ patient days, $P=0.02$ ). Similarly, the incidence of HAls caused by CRAB in intervention group was $19.75 \%$ lower than that in pre-intervention group $(6.0,95 \% \mathrm{Cl} 4.4-7.6$ vs. $7.5,95 \% \mathrm{Cl} 5.7-9.3$ cases / 1,000 patient days, $P=0.24$ ). The DID model analysis showed that $\mathrm{CHG}$ bathing reduced the incidence of CRAB- and CRPA-caused infections in ICU by 1.56 and 2.15 cases/1,000 patient days, respectively, $\mathrm{P}<0.01$, and bathing of every 38 patients $(95 \% \mathrm{Cl}, 21-268)$ and 39 patients $(95 \% \mathrm{Cl}, 24-$ 110) were able to prevent one case of HAls of CRAB and CRPA, respectively. However, CHG bathing showed no effect on MRSA, VRE and CRE ( $P>0.05)$.

Conclusion: Daily bathing with $2 \% \mathrm{CHG}$-impregnated wipes can reduce HAls caused by CRAB and CRPA, while it is not effective for the prevalence of infections caused by MRSA, VRE, and CRE.

\section{Background}

In recent years, the prevalence of healthcare-associated infections (HAls) caused by multidrug-resistant organisms (MDROs) has become seriously, which often causes severe consequences such as prolonged length of stay and increased mortality, and has been listed as a serious threat by the World Health Organization ${ }^{[1,2]}$. MDROs commonly seen in healthcare settings include carbapenem-resistant Acinetobacter baumannii (CRAB), carbapenem-resistant Pseudomonas aeruginosa (CRPA), carbapenemresistant Enterobacteriaceae(CRE), methicillin-resistant Staphylococcus aureus (MRSA), and vancomycin-resistant enterococci (VRE). In order to prevention and control the infections caused by these MDROS, many measures, including hand hygiene, surveillance, contact precaution, isolation, and environment cleaning, have been widely adopted and been recommended by guidelines ${ }^{[3-5]}$.

Daily bathing with $2 \%$ or $4 \%$ chlorhexidine gluconate (CHG) has been considered as an important measure to prevent and control MDROs infections in ICU by decontamination of skin colonization, 
although there is an ongoing controversy about the impact of this procedure ${ }^{[6-8]}$. In vitro experiments have shown that CHG has broad antibacterial activity against Gram-positive and Gram-negative bacteria, and the bacteriostatic effect on the human skin bacteria lasts for 24 hours $^{[9]}$. A number of studies have shown that daily bathing by $\mathrm{CHG}$ wipes in ICU can reduce HAls caused by MRSA and VRE ${ }^{[10-12]}$. However, there is less evidence for CHG bathing on CRAB, CRPA, and CRE. One before-after study has showed slight reduction of infections caused by the multidrug-resistant Acinetobacter baumannii and Pseudomonas aeruginosa ${ }^{[13]}$, while two other studies have exhibited unsatisfactory results ${ }^{[6,14]}$. To assess the effectiveness of CHG bathing in ICU, especially in CRAB and CRPA endemic areas, we therefore conducted this study.

\section{Data And Methods}

\subsection{Study Design}

A before-after study with parallel controls was conducted in a teaching hospital with 4,300 beds in Mainland China. Routine prevention and control measures was conducted before July 2016 while strengthened procedures were employed since then in the whole hospital. In addition, daily bathing with $2 \%$ CHG-impregnated wipes was performed in a general ICU with 50 beds from July 1 to December 31, 2016. In order to masking the influence of seasonal fluctuations on the incidence of HAls of MDROs, patients who admitted to ICU since July to December 2015 were selected as a control group (preintervention group). Meanwhile, 57 non-ICU wards in the hospital which had MDROs infections during the same period were used as parallel controls. Besides daily $\mathrm{CHG}$ bathing, all of the measures in these wards were the same as the ICU. The net effect of $\mathrm{CHG}$ was evaluated by the difference in difference model (DID). The study design is shown in Fig. 1.

\subsection{Inclusion and exclusion criteria}

All of the patients older than 18 years admitted to the general ICU were included. Patients were excluded if they are 1) younger than 18 years; 2) patients with trauma or skin ulceration when stay in ICU; 3) patients stay in ICU less than 48 hours.

\subsection{Intervention Procedure}

\subsubsection{Daily bathing with $2 \%$ CHG-impregnated wipes}

In June 2016, all ICU nurses received training on the standardized procedure of bathing patients using $2 \%$ CHG-impregnated wipes. From July to December 2016, trained nurses performed daily bathing using $2 \%$ CHG-impregnated wipes (Guardwell disposable care wipes, produced by Sichuan Yingnaide Medical Technology Co., Ltd.) for all newly-admitted ICU patients who met the inclusion criteria until they left the ICU. Each pack of the wipes has a total of eight pieces. The wipes were placed in an incubator at the constant temperature of $40^{\circ} \mathrm{C}$ for heat treatment before use. The bathing steps were carried out in accordance with the recommended sequence of Agency for Healthcare Research Quality ${ }^{[15]}$, briefly as 
follows: Cloth 1: Neck, shoulders and chest; Cloth 2: Both arms, hands, web spaces, and axilla; Cloth 3: Abdomen and then groin/perineum; Cloth 4: Right leg, foot, and web spaces; Cloth 5: Left leg, foot, and web spaces; Cloth 6: Back of neck, back and then buttocks. Use additional CHG bathing cloths, if necessary, to thoroughly cleanse the body. The infection control link nurse in the ICU supervised the compliance of the bathing.

\subsubsection{Strengthened measures for MDROs}

"MDRO prevention and control policy" had been revised and implemented in the whole hospital since July 2016. The newly added strengthened measures included four contents: 1) MDROs' information was immediately informed to the doctors of the corresponding patients when detected by the microbiology lab, and an isolation tag was automatically added on the hospital information system (HIS); 2) The summary of MDRO infections (the total patient numbers, the bed number for each patient, and the species and resistance type of each MDRO) in the unit was briefed in the daily morning shift meeting; 3 ) The number of doctors perform medical rounds in the room was not allowed to exceed 3 for patients with MDRO infections, and only a single designated nurse is in charge of the care for patients with MDRO infections during each shift; 4) a single room was preferred for placing patients with MDRO infections whenever possible. When single rooms are not available, the patients are settled in the corner area (rather than in the middle of multiple beds) of the room.

\subsection{Definitions}

The diagnostic criteria for HAls were the Diagnostic Standards for HAls published by the Ministry of Health in 2001 ${ }^{[16]}$. The MDROs in this study include CRAB, CRPA, CRE, VRE, and MRSA.

\subsection{Data Collection}

Demographic and clinical data of patients were collected, including gender, age, ethnicity, invasive procedures, medical orders, underlying disease, APACHE II score, and length of stay.

\subsection{Outcome Indices}

The incidence density of HAls of targeted multidrug-resistant bacteria (cases/1,000 patient days) in ICU was used as the primary evaluation index. Other indices included the number needed to treat (NNT) and its $95 \%$ confidence interval.

\subsection{Statistics}

For descriptive analyses, qualitative data are expressed in terms of frequency (rate), and quantitative data are expressed as the median (interquartile range). Qualitative data were analyzed by the chi-square test or Fisher's exact test. Quantitative data which were skewed based on the normality test and were analyzed using the Mann-Whitney U test. Using intention-to-treat (ITT) analysis, all patients who met the inclusion criteria in the ICU ward during the intervention period were analyzed as being given the CHG sponge bath. Poisson's test was used to compare the incidence density of multidrug-resistant bacterial infections before and after intervention in ICU. 
The difference in difference model (DID) of the fixed effect estimation method was used to quantitatively analyze the effect of daily $\mathrm{CHG}$ sponge bath on reducing multidrug-resistant bacterial infection. The basic setting of the DID model is

$$
y_{i t}=\beta_{0}+\beta_{1} \text { group }_{i} \cdot \text { time }_{t}+\beta_{2} \text { group }_{i}+\gamma \text { time }_{t}+\varphi \mathrm{Xit}+\varepsilon_{i t}(i=1, \ldots, 46 ; t=1,2)
$$

where group $_{i}$ is the dummy variable for group (if $\operatorname{group}_{i}=1$, then it belongs to the experimental group; if group $_{i}=0$, it belongs to the control group), which characterizes the difference of the experimental group and the control group themselves (that is, even without sponge bath, this difference still exists.); time $e_{t}$ is the dummy variable for time (if $\mathrm{t}=2$ and time $_{t}=1$, it is in the intervention period; if $\mathrm{t}=1$ and time $_{t}=0$, it is in the pre-intervention period), and even without sponge bath, this time tendency still exists; group time $_{t}$ is an interaction term, and its coefficient $\beta_{1}$ is a DID estimation value that measures the effect of $\mathrm{CHG}$. To assess whether the results are robust, other explanatory variables, $X_{i t}$, were introduced for sensitivity analysis, including mortality, average bed turnover frequency, bed occupancy rate, average bed occupied days, hand hygiene accuracy rate, and hand hygiene compliance.

Microsoft-Excel 2016 was used to build a database for data processing and management. Statistical analysis and the DID method were processed using Stata 9.2 (Stata / SE 9. 2 Stata Corporation, College Station, TX, USA) software.

\subsection{Ethics Approval}

The study was approved by the Ethics Committee of Sichuan University. Bathing had been regarded as part of the normal care for ICU patients and inform consents were waived.

\section{Results}

\subsection{Characteristics of ICU Patients of the Pre-Intervention and Intervention Period}

From July 1 to December 31, 2016, a total of 1,009 patients were admitted to the general ICU. The compliance with the daily CHG bathing was $81.96 \%(827 / 1,009)$. Both of the demographic characteristics and underlying diseases of ICU patients during the intervention and pre-intervention period are comparable (Table 1). The occupancy rate of beds and the compliance of basic infection control measures including hand hygiene and environment cleaning are also comparable (Table 2). 
Table 1

Characteristics of patients with or without chlorhexidine bathing

\begin{tabular}{|c|c|c|c|}
\hline Characteristic & $\begin{array}{l}\text { Without Chlorhexidine ( } \mathrm{n} \\
=833 \text { ) }\end{array}$ & $\begin{array}{l}\text { With Chlorhexidine }(\mathrm{n}= \\
\text { 1009) }\end{array}$ & $\begin{array}{l}P \\
\text { Value }\end{array}$ \\
\hline Age, median (interquartile range) & $58(45-69)$ & $58(45-68)$ & 0.64 \\
\hline Male sex & $550(66.0)$ & $638(63.2)$ & 0.21 \\
\hline Han nationality & $799(96.3)$ & 958 (94.9) & 0.81 \\
\hline Indwelling CVC & $368(44.2)$ & 487 (48.3) & 0.08 \\
\hline Mechanical ventilation & 735 (88.2) & $862(85.4)$ & 0.08 \\
\hline Indwelling urinary catheter & 799 (95.9) & $960(95.1)$ & 0.43 \\
\hline Hypertension & $200(24)$ & $240(23.8)$ & 0.91 \\
\hline Diabetes & $113(13.6)$ & $139(13.8)$ & 0.90 \\
\hline Pancreatitis & $78(9.4)$ & $95(9.4)$ & 0.97 \\
\hline Respiratory failure & $194(23.3)$ & $211(20.9)$ & 0.22 \\
\hline Renal insufficiency & $104(12.5)$ & $118(11.7)$ & 0.60 \\
\hline AIDS & $1(0.1)$ & $0(0)$ & 0.27 \\
\hline Malignant tumor & $312(37.5)$ & $350(34.7)$ & 0.22 \\
\hline Hematonosis & $18(2.2)$ & $11(1.1)$ & 0.07 \\
\hline COPD & $37(4.4)$ & $41(4.1)$ & 0.69 \\
\hline Tuberculosis & $10(1.2)$ & $28(2.8)$ & 0.02 \\
\hline $\begin{array}{l}\text { APACHE II score, median } \\
\text { (interquartile range) }\end{array}$ & $15(9-22)$ & $14(8-21)$ & 0.13 \\
\hline $\begin{array}{l}\text { Length of stay, median (interquartile } \\
\text { range) }\end{array}$ & $21(13-32)$ & $20(13-33)$ & 0.67 \\
\hline \multicolumn{4}{|c|}{$\begin{array}{l}\text { Abbreviations: CVC, Central venous catheter; APACHE II, Acute Physiology and Chronic Health } \\
\text { Evaluation II; AIDS, Acquired immune deficiency syndrome; COPD, Chronic obstructive pulmonary } \\
\text { disease. }\end{array}$} \\
\hline
\end{tabular}


Table 2

Characteristics of units comparing the baseline and intervention periods

\begin{tabular}{|llll|}
\hline Unit characteristics & Baseline period & Intervention period & PValue \\
\hline Average number of open beds & 50 & 50 & $/$ \\
\hline Mean length of stay (d) & 26.2 & 26.7 & $/$ \\
\hline Occupancy rate of beds (\%) & $94.6(8700 / 9200)$ & $94.3(8672 / 9200)$ & 0.37 \\
\hline Hand hygiene compliance (\%) & $89.3(67 / 75)$ & $94.6(122 / 129)$ & 0.17 \\
\hline Environment cleaning compliance (\%) & $94.0(63 / 67)$ & $96.7(269 / 280)$ & 0.46 \\
\hline The Combined Effect Of Strengthened Prevention and \\
\hline Thtrol Measures and CHG Bathing & &
\end{tabular}

The incidence density of HAls caused by CRPA during the intervention period was significantly decreased compared with that during the pre-intervention period $(2.5$ [95\% $\mathrm{Cl} 1.6-3.8]$ cases/1,000 patient days vs. $4.6[95 \% \mathrm{Cl} 3.3-6.3]$ cases/ 1,000 patient days, $P=0.02$ ). HAls caused by CRAB decreased by $19.75 \%$, although the difference was not statistically significant $(6.0$ [95\% $\mathrm{Cl} 4.4-7.6]$ cases / 1,000 patient days vs. $7.5[95 \% \mathrm{Cl} 5.7-9.3]$ cases / 1,000 patient days, $\mathrm{P}=0.24)$. On the basis of strengthened prevention and control measures, application of $2 \%$ CHG bathing every $38(95 \% \mathrm{Cl}, 21-268)$ patients would prevent 1 case of HAls caused by CRAB, and application of CHG to bath every $39(95 \% \mathrm{Cl}, 24-110)$ patients would prevent one case of HAls caused by CRPA. However, the HAls rate caused by MRSA, VRE, and CRE were not decreased significantly in the intervention (Table 3). The incidence density of HAls caused by MRSA, VRE and CRE during the pre-intervention period was 0.2 [95\% $\mathrm{Cl} 0-0.8], 0.1$ [95\% $\mathrm{Cl} 0-0.6$ ] and 0.5 [95\% $\mathrm{Cl} 0.1-1.2]$ cases $/ 1,000$ patient days separately. And The incidence density of HAls caused by MRSA, VRE and CRE during the intervention period was 0.2 [95\% $\mathrm{Cl} 0-0.8$ ], 0.1 [95\% $\mathrm{Cl} 0-0.6$ ] and 0.6 [95\% $\mathrm{Cl} 0.2-1.3]$ cases $/ 1,000$ patient days separately. 
Table 3

HAls caused by MDROs comparing the baseline and intervention periods in the ICU

\begin{tabular}{|llllll|}
\hline \multirow{2}{*}{ MDROs } & \multicolumn{2}{l}{ Baseline period } & \multicolumn{2}{l}{ Intervention period } & \multirow{2}{*}{ Pvalue } \\
\cline { 2 - 5 } & Cases & Incidence density $(95 \%$ Cl) & Cases & Incidence density (95\% Cl) & \\
\hline CRAB & 65 & $7.5(5.7-9.3)$ & 52 & $6.0(4.4-7.6)$ & 0.24 \\
\hline CRPA & 40 & $4.6(3.3-6.3)$ & 22 & $2.5(1.6-3.8)$ & 0.02 \\
\hline MRSA & 2 & $0.2(0-0.8)$ & 2 & $0.2(0-0.8)$ & 1.00 \\
\hline VRE & 1 & $0.1(0-0.6)$ & 1 & $0.1(0-0.6)$ & 1.00 \\
\hline CRE & 4 & $0.5(0.1-1.2)$ & 5 & $0.6(0.2-1.3)$ & 1.00 \\
\hline Total & 112 & $12.9(10.5-15.3)$ & 82 & $9.5(7.4-11.5)$ & 0.03 \\
\hline The unit of incidence density is cases per 1,000 inpatient days. & \\
\hline $\begin{array}{l}\text { Abbreviations: HAls, Healthcare associated infections; MDROs, Multi-drug resistant organisms; CRAB, } \\
\text { Carbapenem-resistant Acinetobacter baumannii; BSI, Bloodstream infection; CRPA, Carbapenem- } \\
\text { resistant Pseudomonas aeruginosa; MRSA, Methicillin resistant Staphylococcus aureus; VRE, } \\
\text { Vancomycin-resistant Enterococci; CRE, Carbapenem-resistant Enterobacteriaceae. }\end{array}$ \\
\hline
\end{tabular}

\subsection{The Net Effect of 2\% CHG Bathing}

There was no statistically significant difference in compliance to the strengthened prevention and control measure between ICU and the general wards (644 observations, $94.57 \%$ vs. 664 observations, $92.32 \%, P$ $=0.101)$. After introduction of the parallel control, the DID model showed that CHG bathing significantly reduced the incidence density of HAls caused by CRAB and CRPA by 1.56 and 2.15 cases/1,000 patient days, respectively $(P<0.01)$, and appeared to reduce the incidence density of HAls caused by MRSA by 0.05 cases $/ 1,000$ patient days but without statistical significance $(P=0.92)$, while showing no effect on VRE and CRE (Table 4).

Table 4

DID model estimation results of HAls caused by MDROs

\begin{tabular}{|lllll|}
\hline MDRO & Diff-in-Diff & S. Err. & $\boldsymbol{I} \boldsymbol{t} \mid$ & $\boldsymbol{P}$ value \\
\hline CRAB & -1.555 & 0.107 & 14.5 & $<0.01$ \\
\hline CRPA & -2.152 & 0.033 & 65.4 & $<0.01$ \\
\hline MRSA & -0.053 & 0.536 & 0.1 & 0.92 \\
\hline VRE & 0.029 & 0.017 & 1.7 & 0.10 \\
\hline CRE & 0.053 & 0.045 & 1.2 & 0.25 \\
\hline Total & -3.617 & 0.501 & 7.23 & $<0.01$ \\
\hline
\end{tabular}


After the inclusion of the covariates that may affect the results, the sensitivity analysis of the model (Table 5) shows that the results of the statistical differences do not change. This indicates that the results are robust.

Table 5

Sensitivity analysis of DID model

\begin{tabular}{|lllll|}
\hline MDRO & Diff-in-Diff & S. Err. & It & $P$ value \\
\hline CRAB & -1.538 & 0.188 & 8.2 & $<0.01$ \\
\hline CRPA & -2.235 & 0.090 & 24.9 & $<0.01$ \\
\hline MRSA & -0.263 & 1.477 & 0.2 & 0.86 \\
\hline VRE & 0.030 & 0.033 & 0.9 & 0.37 \\
\hline CRE & 0.218 & 0.190 & 1.2 & 0.26 \\
\hline Total & -3.030 & 1.042 & 2.9 & $<0.01$ \\
\hline $\begin{array}{l}\text { Note: Included covariates are mortality, average bed turnover frequency, bed occupancy rate, average } \\
\text { bed occupied days, hand hygiene accuracy rate, and hand hygiene compliance. }\end{array}$ & \\
\hline
\end{tabular}

\subsection{Adverse Reactions}

All patients who received daily $2 \%$ CHG bathing did not develop any adverse reactions such as skin redness and allergies.

\section{Discussion}

HAls caused by MDRO have always been the focus of infection prevention and control. Before July 2016, a series of prevention and control measures were practiced in our hospital according to the Guidelines ${ }^{[5]}$, including hand hygiene, environment cleaning and disinfection, monitoring and feedback, education for healthcare workers, and patient isolation. However, these measures had little effect as the occurrence of MDRO infections was still high and showed no a decreasing trend. Since July 1, 2016, we implemented strengthened measures on the basis of previous measures according to the actual conditions of the hospital and introduced the CHG bathing in ICU. Our study showed that the application of bathing with $2 \%$ CHG wipes reduced CRAB and CRPA infection in the ICU by 1.56 and 2.15 cases/1,000 patient days, respectively, similar to the studies before ${ }^{[13,17]}$. However, Ruiz ${ }^{[13]}$ only performed bath for patients who used mechanical ventilation and those who colonized with MDRO, and Chung ${ }^{[17]}$ only evaluated the effect on CRAB. Because this study was performed in the real world, the ICU had taken strengthened prevention and control measures while we were performing the daily bathing using $2 \% \mathrm{CHG}$ wipes, and thereby the comparison with ICU itself before and after intervention can only reflect the combined effect of these two measures. Therefore, we selected 57 non-ICU wards that implemented the same strengthened measures and had a compliance rate similar to that of the ICU as parallel control, and used the DID model to estimate the net effect of CHG. The DID model is an econometric method which widely 
used in the fields of economics and sociology and has been used in the field of infection prevention and control before ${ }^{[18]}$. This study applied this model to the semi-experimental research of prevention and control of MDROs, which effectively evaluated the control effect of $2 \%$ CHG daily bath on MDROs.

Furthermore, one study ${ }^{[10]}$ have shown that using $\mathrm{CHG}$ bathing to decolonize every 54 patients can prevent one case of bloodstream infection caused by MRSA. However, few studies have demonstrated the efficacy of daily $2 \% \mathrm{CHG}$ bathing on CRAB and CRPA infection. This study shows that on the basis of strengthened prevention and control measures, $2 \% \mathrm{CHG}$ bathing for every 38 patients can prevent one case of HAls caused by CRAB, and $2 \%$ CHG bathing for every 39 patients can prevent one case of HAls caused by CRPA, demonstrating good cost-effectiveness. Unlike the results of other studies ${ }^{[10-12]}$, our study did not find that CHG bathing could reduce HAls caused by MRSA, VRE and CRE. This may be due to the fewer cases of HAls caused by MRSA, VRE and CRE in the ICU during the study period, suggesting that in the low-prevalence areas, general decolonization using $2 \% \mathrm{CHG}$ wipe has limited value.

In addition, in this study we did not employ the routine time-series design but decided to choose the same time period of the previous year as a pre-intervention control. This is mainly due to the fact that some studies have shown that seasonal factors may affect the incidence of HAls caused by MDROs ${ }^{[19-22]}$, especially in the summer when the infections caused by Gram-negative bacilli may increase significantly. Therefore, our choice of the same time period of the previous year would well mask this confounding factor.

This study also has some limitations. First, it is a single-center study which was carried out in an area where CRAB and CRPA are notably prevalent, which limits the extrapolation of the results; however, it has significance for areas with high CRAB and CRPA prevalence. Second, we did not conduct an active surveillance screening of CRAB and CRPA and could not evaluate the effect of CHG bathing on colonized bacteria. Studies have confirmed that $\mathrm{CHG}$ bathing can reduce the colonization rate of MDRO on the body surface, and this study shows that CHG bathing can reduce HAls caused by CRAB and CRPA, which may also be due to the reduction of body surface colonized bacteria by the entire body bath, thereby reducing the risk of HAls caused by these bacteria. Finally, as the ICU ward adopted two measures simultaneously during the intervention, the comparison before and after the intervention could not reflect the role of CHG alone. Therefore, we resorted to the DID model to introduce the common wards as a control to assess the net effect of $\mathrm{CHG}$.

\section{Conclusion}

In conclusion, this study demonstrates that daily bathing for ICU patients with $2 \%$ CHG wipes can reduce HAls caused by CRAB and CRPA in endemic areas. However, in the future, multicenter clustered randomized controlled studies are required to evaluate the prevention and control the effect of a daily CHG sponge bath against multidrug-resistant Gram-negative bacteria.

\section{Declarations}




\section{Ethics approval and consent to participate}

The study was approved by the Ethics Committee of Sichuan University. Bathing had been regarded as part of the normal care for ICU patients and inform consents were waived.

\section{Consent for publication}

Not applicable.

\section{Availability of data and materials}

Not applicable.

\section{Competing interests}

The authors declare that they have no competing interests.

\section{Funding}

This work was supported by grants from the Science and Technology Department of Sichuan Province区 P.R. China (grant number 2017ZR0170).

\section{Authors' contributions}

Wenzhi Huang, Zhiyong Zong and Wei Zhang contributed to study conception and design. Wenzhi Huang, Fu Qiao and Lin Cai contributed to acquisition of data. Wenzhi Huang analyzed and interpreted data. Wenzhi Huang, Zhiyong Zong and Wei Zhang drafted the manuscript. All authors revised the manuscript for important intellectual content. All authors read and approved the final manuscript.

\section{Acknowledgments}

We would like acknowledge the physicians and allied health staff of the ICU and the Department of Electronic Medical Record system of West China Hospital.

\section{References}

1. Global Priority List of Antibiotic-resistant. Bacteria to Guide Research, Discovery, and Development of New Antibiotics.[J]. 2019.

2. Rello J, Kalwaje EV, Lagunes $L$, et al. A global priority list of the TOp TEn resistant Microorganisms (TOTEM) study at intensive care: a prioritization exercise based on multi-criteria decision analysis[J]. Eur J Clin Microbiol Infect Dis. 2019;38(2):319-23.

3. Guidelines for the Prevention and Control of Carbapenem-Resistant Enterobacteriaceae. Acinetobacter baumannii and Pseudomonas aeruginosa in Health Care Facilities[M]. Geneva: World Health Organization; 2017. 
4. Siegel JD, Rhinehart $E$, Jackson $M$, et al. Management of multidrug-resistant organisms in health care settings, 2006[J]. Am J Infect Control. 2007;35(10 Suppl 2):165-93.

5. Tacconelli E, Mazzaferri F, de Smet AM, et al. ESCMID-EUCIC clinical guidelines on decolonization of multidrug-resistant Gram-negative bacteria carriers[J]. Clin Microbiol Infect. 2019;25(7):807-17.

6. Noto MJ, Domenico HJ, Byrne DW, et al. Chlorhexidine bathing and health care-associated infections: a randomized clinical trial[J]. JAMA. 2015;313(4):369-78.

7. Huang SS, Septimus E, Kleinman K, et al. Chlorhexidine versus routine bathing to prevent multidrugresistant organisms and all-cause bloodstream infections in general medical and surgical units (ABATE Infection trial): a cluster-randomised trial[J]. Lancet. 2019;393(10177):1205-15.

8. Huang SS. Chlorhexidine-based decolonization to reduce healthcare-associated infections and multidrug-resistant organisms (MDROs): who, what, where, when, and why?[J]. J Hosp Infect. 2019;103(3):235-43.

9. Popovich KJ, Lyles R, Hayes R, et al. Relationship between chlorhexidine gluconate skin concentration and microbial density on the skin of critically ill patients bathed daily with chlorhexidine gluconate[J]. Infect Control Hosp Epidemiol. 2012;33(9):889-96.

10. Huang SS, Septimus E, Kleinman K, et al. Targeted versus universal decolonization to prevent ICU infection[J]. N Engl J Med. 2013;368(24):2255-65.

11. Frost SA, Alogso MC, Metcalfe $L$, et al. Chlorhexidine bathing and health care-associated infections among adult intensive care patients: a systematic review and meta-analysis[J]. Crit Care. 2016;20(1):379.

12. Climo MW, Yokoe DS, Warren DK, et al. Effect of daily chlorhexidine bathing on hospital-acquired infection[J]. N Engl J Med. 2013;368(6):533-42.

13. J R, P R, E V, et al. Daily bathing strategies and cross-transmission of multidrug-resistant organisms: Impact of chlorhexidine-impregnated wipes in a multidrug-resistant gram-negative bacteria endemic intensive care unit[J]. Am J Infect Control, 2017,45(10):1069-1073.

14. Boonyasiri A, Thaisiam P, Permpikul C, et al. Effectiveness of Chlorhexidine Wipes for the Prevention of Multidrug-Resistant Bacterial Colonization and Hospital-Acquired Infections in Intensive Care Unit Patients: A Randomized Trial in Thailand[J]. Infect Control Hosp Epidemiol. 2016;37(3):245-53.

15. Agency For Healthcare Research and Quality. Universal ICU Decolonization: An Enhanced Protocol[J]. AHRQ Publication No. 13-0052-EF., 2013.

16. Liu JY, Wu YH, Cai M, et al. Point-prevalence survey of healthcare-associated infections in Beijing, China: a survey and analysis in 2014[J]. J Hosp Infect. 2016;93(3):271-9.

17. Chung YK, Kim JS, Lee SS, et al. Effect of daily chlorhexidine bathing on acquisition of carbapenemresistant Acinetobacter baumannii (CRAB) in the medical intensive care unit with CRAB endemicity[J]. Am J Infect Control. 2015;43(11):1171-7.

18. Halpin HA, McMenamin SB, Simon LP, et al. Impact of Participation in the California HealthcareAssociated Infection Prevention Initiative on Adoption and Implementation of Evidence-Based 
Practices for Patient Safety and Health Care-Associated Infection Rates in a Cohort of Acute Care General Hospitals. [J]. Am J Infect Control. 2013;41(4):307-11.

19. Ramos GP, Rocha JL, Tuon FF. Seasonal humidity may influence Pseudomonas aeruginosa hospitalacquired infection rates[J]. Int $J$ Infect Dis. 2013;17(9):e757-61.

20. Psoter KJ, De Roos AJ, Wakefield J, et al. Season is associated with Pseudomonas aeruginosa acquisition in young children with cystic fibrosis[J]. Clin Microbiol Infect. 2013;19(11):E483-9.

21. Chazan B, Colodner R, Edelstein $\mathrm{H}$, et al. Seasonal variation in Escherichia coli bloodstream infections in northern Israel[J]. Clin Microbiol Infect. 2011;17(6):851-4.

22. Perencevich EN, McGregor JC, Shardell M, et al. Summer Peaks in the Incidences of Gram-Negative Bacterial Infection Among Hospitalized Patients[J]. Infect Control Hosp Epidemiol. 2008;29(12):1124-31.

\section{Figures}

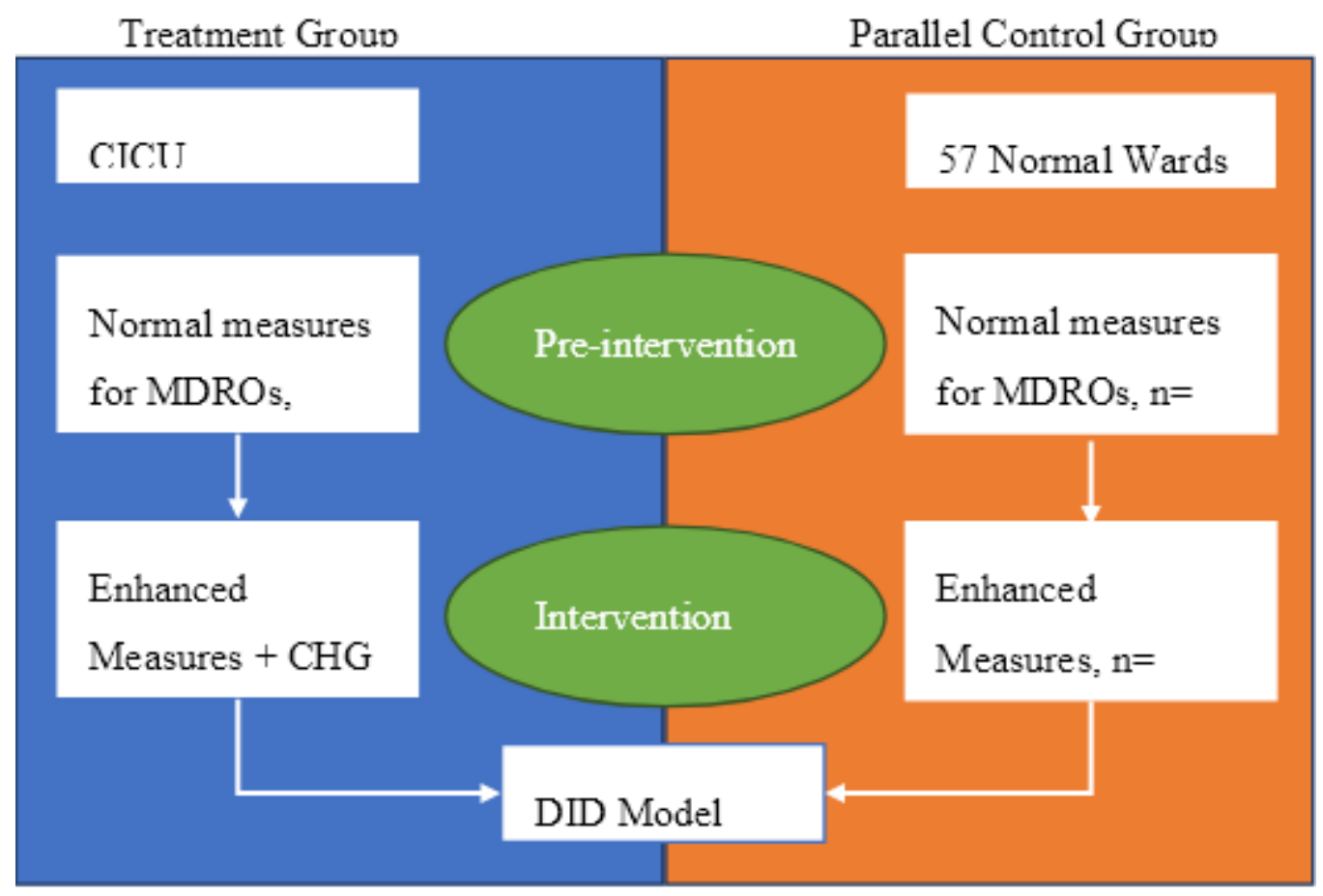

Figure 1. Study design

\section{Figure 1}

Study design 